TRANSACTIONS OF THE

AMERICAN MATHEMATICAL SOCIETY

Volume 349, Number 10, October 1997, Pages 4085-4105

S 0002-9947(97)01889-8

\title{
THE SZEGÖ CURVE, ZERO DISTRIBUTION AND WEIGHTED APPROXIMATION
}

\author{
IGOR E. PRITSKER AND RICHARD S. VARGA
}

ABSTRACT. In 1924, Szegő showed that the zeros of the normalized partial sums, $s_{n}(n z)$, of $e^{z}$ tended to what is now called the Szegö curve $S$, where

$$
S:=\left\{z \in \mathbb{C}:\left|z e^{1-z}\right|=1 \text { and }|z| \leq 1\right\} .
$$

Using modern methods of weighted potential theory, these zero distribution results of Szegö can be essentially recovered, along with an asymptotic formula for the weighted partial sums $\left\{e^{-n z} s_{n}(n z)\right\}_{n=0}^{\infty}$. We show that $G:=\operatorname{Int} S$ is the largest universal domain such that the weighted polynomials $e^{-n z} P_{n}(z)$ are dense in the set of functions analytic in $G$. As an example of such results, it is shown that if $f(z)$ is analytic in $G$ and continuous on $\bar{G}$ with $f(1)=0$, then there is a sequence of polynomials $\left\{P_{n}(z)\right\}_{n=0}^{\infty}$, with $\operatorname{deg} P_{n} \leq n$, such that

$$
\lim _{n \rightarrow \infty}\left\|e^{-n z} P_{n}(z)-f(z)\right\|_{\bar{G}}=0,
$$

where $\|\cdot\|_{\bar{G}}$ denotes the supremum norm on $\bar{G}$. Similar results are also derived for disks.

\section{INTRODUCTION}

Let $\left\{s_{n}(z):=\sum_{k=0}^{n} \frac{z^{k}}{k !}\right\}_{n=0}^{\infty}$ denote the familiar partial sums of the exponential function $e^{z}$. In his seminal paper of 1924, Szegő [12] showed that the normalized partial sum $s_{n}(n z)$ satisfies the following equation:

$$
e^{-n z} s_{n}(n z)=1-\frac{\sqrt{n}}{\tau_{n} \sqrt{2 \pi}} \int_{0}^{z}\left(\zeta e^{1-\zeta}\right)^{n} d \zeta, \quad z \in \mathbb{C}
$$

for any $n \geq 1$, where from Stirling's asymptotic series formula (cf. Henrici [6, p.

$$
\tau_{n}:=\frac{n !}{n^{n} e^{-n} \sqrt{2 \pi n}} \approx 1+\frac{1}{12 n}+\frac{1}{288 n^{2}}-\frac{139}{51840 n^{3}}+\cdots, \quad n \rightarrow \infty,
$$

so that $\lim _{n \rightarrow \infty} \tau_{n}=1$.

One of the major items in Szegö's work was the analytic function $\varphi(z):=z e^{1-z}$, which appears in the integrand of (1.1). Szegö introduced what is now called the Szegö curve $\{z \in \mathbb{C}:|\varphi(z)|=1\}$, and he showed that it divides the complex plane

Received by the editors March 30, 1996.

1991 Mathematics Subject Classification. Primary 30E10; Secondary 30C15, 31A15, 41A30.

Key words and phrases. Szegö curve, weighted polynomials, weighted energy problem, extremal measure, logarithmic potential, balayage, modified Robin constant. 


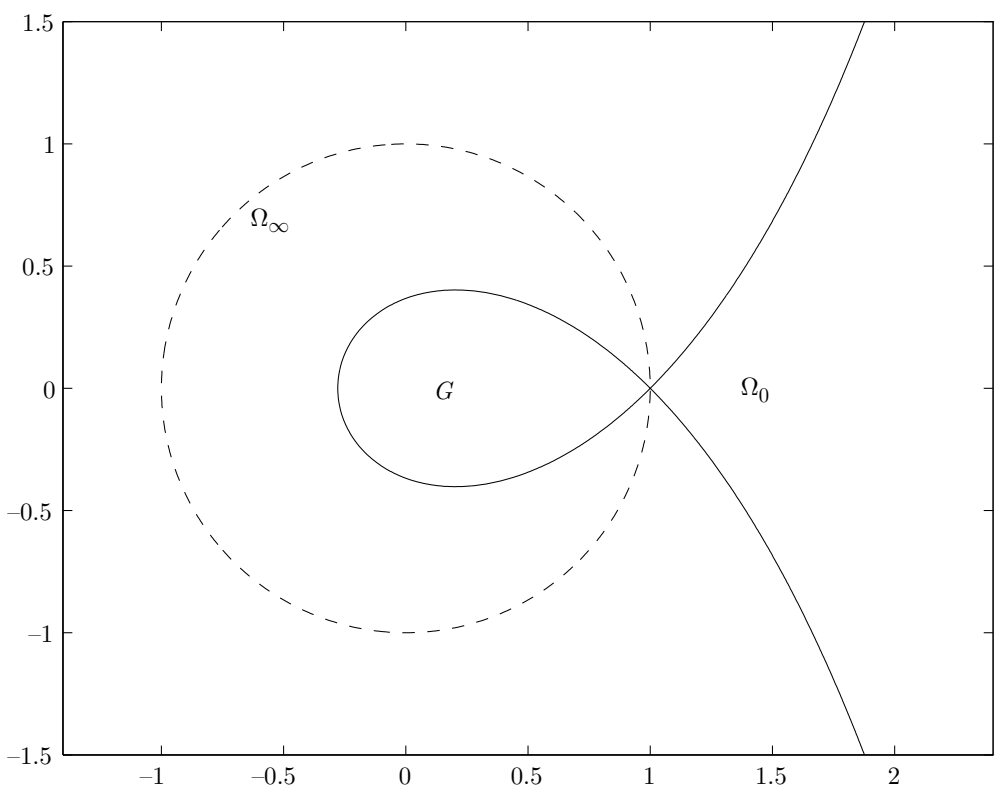

Figure 1. The Szegő curve and the associated domains

$\mathbb{C}$ into three domains. One of them is the bounded domain $G$, contained in the unit disk $D:=\{z \in \mathbb{C}:|z|<1\}$, whose boundary consists of that part of the Szegö curve, namely

$$
S:=\left\{z \in \mathbb{C}:\left|z e^{1-z}\right|=1 \text { and }|z| \leq 1\right\},
$$

which is contained in the closed unit disk. We remark that $S$ is a piecewise analytic Jordan curve with one corner point at $z=1$. Szego" [12] proved that $G$, in the $z$ plane, is mapped conformally onto the unit disk $D$, in the $w$-plane, by the function $w=\varphi(z)$, and that the unbounded domains, also determined from the Szegö curve, are given by $\Omega_{0}:=\{z:|\varphi(z)|<1,|z|>1\}$ and $\Omega_{\infty}:=\{z:|\varphi(z)|>1\}$. This is shown in Figure 1.

A main result of Szegö [12] is the asymptotic zero distribution of the normalized partial sums $\left\{s_{n}(n z)\right\}_{n=1}^{\infty}$. Specifically, Szego proved that $\zeta$ is an accumulation point of the zeros of $\left\{s_{n}(n z)\right\}_{n=1}^{\infty}$ if and only if $\zeta \in S$. Moreover, the images of zeros of $s_{n}(n z)$, under the mapping $w=\varphi(z)$, are asymptotically uniformly distributed in angle (see [12]), near the unit circle $T=\{w \in \mathbb{C}:|w|=1\}$, as $n \rightarrow \infty$.

Using modern methods of weighted potential theory (cf. Saff and Totik [11]), we shall essentially recover, in Section 2 of this paper, the zero distribution results of Szegö, together with an asymptotic formula for the weighted normalized partial sums $\left\{e^{-n z} s_{n}(n z)\right\}_{n=0}^{\infty}$.

As shown below in Proposition 3.1, a consequence of (1.1) is that $e^{-n z} s_{n}(n z)$ converges to $f(z) \equiv 1$, locally uniformly in $G$ (i.e., uniformly on compact subsets of $G$ ). This naturally leads to the question of the possibility of uniform approximation of any function, analytic in $G$, by weighted polynomials $\left\{e^{-n z} P_{n}(z)\right\}_{n=0}^{\infty}$, where $P_{n}$ is a complex polynomial with $\operatorname{deg} P_{n} \leq n$ for each $n \geq 0$. This type of problem evolved from Lorentz's approximation by "incomplete polynomials" [8] 
on the real line, and it has been developed into the general theory of approximation with varying weights (for excellent treatments of the theory and history of weighted approximation, see Saff and Totik [11] and Totik [13]). However, Szegő's equation (1.1) can be considered as the historical predecessor of all such approximation problems. Furthermore, as we shall see, (1.1) opens the door to a special weighted approximation of analytic functions in the complex plane, as opposed to weighted approximation of functions on the real line. We remark that weighted approximation in the complex plane has, with the exception of the recent paper by Borwein and Chen [1], received far less attention in the current approximation theory literature. In contrast, we derive here various new results on the density of the weighted polynomials $\left\{e^{-n z} P_{n}(z)\right\}_{n=0}^{\infty}$ in the set of analytic functions, and we shall also investigate in Section 3 the rates of convergence for special approximating sequences of weighted polynomials.

Section 4 is devoted to a weighted potential problem with a special weight. All proofs of the results stated in Sections 2 and 3 are given in Section 5.

\section{Normalized Partial Sums $s_{n}(n z)$}

With the Szegö curve $S$ of (1.3) and its interior $G$, the harmonic measure at the point $z=0$, with respect to $G$, is defined as the preimage of the normalized arc-length measure on $T:=\{w \in \mathbb{C}:|w|=1\}$, under the mapping $w=\varphi(z)$, where $\varphi(z)=z e^{1-z}$, i.e.,

$$
\omega(0, B, G):=m(\varphi(B \cap S)),
$$

where $d m:=d \theta /(2 \pi)$ on $T$, for any Borel set $B \subset \mathbb{C}$. From $(2.1)$, we see that $\omega(0, \cdot, G)$ is a unit Borel measure which is supported on $S$, i.e., $\omega(0, \mathbb{C}, G)=1$ and $\operatorname{supp} \omega(0, \cdot, G)=S$. For any complex polynomial $P_{n}(z)$ with $\operatorname{deg} P_{n}=n$, we introduce the normalized counting measure of its zeros:

$$
\nu_{n}\left(P_{n}\right):=\frac{1}{n} \sum_{P_{n}\left(z_{i}\right)=0} \delta_{z_{i}},
$$

where $\delta_{z}$ is the unit point mass at $z$ and where all zeros are counted according to their multiplicities. Our results on asymptotic zero distributions are stated in terms of the weak* convergence of measures, i.e., a sequence of Borel measures $\left\{\mu_{n}\right\}_{n=1}^{\infty}$ on $\mathbb{C}$ converges to the measure $\mu$, as $n \rightarrow \infty$, in the weak ${ }^{*}$ topology (written $\mu_{n} \stackrel{*}{\rightarrow} \mu$ ) if

$$
\lim _{n \rightarrow \infty} \int f d \mu_{n}=\int f d \mu,
$$

for any continuous function $f$ on $\mathbb{C}$ having compact support.

The following theorem is, in the large, a restatement of Szegö's results [12]. (For its proof, see Section 5.)

Theorem 2.1. For the normalized partial sums of $e^{z}$, we have

$$
\nu_{n}\left(s_{n}(n z)\right) \stackrel{*}{\rightarrow} \omega(0, \cdot, G), \text { as } n \rightarrow \infty,
$$

and

$$
\lim _{n \rightarrow \infty}\left|e^{-n z} s_{n}(n z)\right|^{1 / n}=|\varphi(z)|, \quad z \in \mathbb{C} \backslash \bar{G},
$$

where the convergence in (2.4) is uniform on compact subsets of $\mathbb{C} \backslash \bar{G}$. 
The result of (2.4) shows that $e^{-n z} s_{n}(n z)$ diverges unboundedly in $\Omega_{\infty}$ (where $|\varphi(z)|>1$ ), and converges to the identically zero function in $\Omega_{0}$ (where $|\varphi(z)|<1$ ), as $n \rightarrow \infty$. It is possible to obtain more precise asymptotics for $e^{-n z} s_{n}(n z)$ than that of (2.4), as is done, for example in Carpenter et al. [3] and [4], but we do not pursue this direction here.

The behavior of the sequence $\left\{e^{-n z} s_{n}(n z)\right\}_{n=0}^{\infty}$ on $\bar{G}$, which is not covered in (2.4) of Theorem 2.1, will be discussed in detail in the next section. Finally, $\|\cdot\|_{E}$ will throughout denote the supremum norm on a set $E$.

\section{Approximation by the Weighted Polynomials $e^{-n z} P_{n}(z)$}

This section concerns the approximation of functions, analytic in $G$, by the weighted polynomials $\left\{e^{-n z} P_{n}(z)\right\}_{n=0}^{\infty}$, where $\operatorname{deg} P_{n} \leq n$. We begin with the approximation of the particular function $f(z) \equiv 1$.

Proposition 3.1. For the weighted normalized partial sums $e^{-n z} s_{n}(n z)$, the following inequality is valid:

$$
\left|e^{-n z} s_{n}(n z)-1\right| \leq \frac{4}{\sqrt{2 \pi n}|z-1|}, \quad z \in \bar{G} \backslash\{1\}, \quad n \geq 1 .
$$

We remark that the inequality of (3.1) of Proposition 3.1, which is in a form useful for our subsequent developments, is closely related to similar results of Szegö $\left[12\right.$, eq. $\left.\left(5^{\prime}\right)\right]$ and $[4$, eq. (2.13)], but is not implied by these results.

The estimate (3.1) shows that the function $f(z) \equiv 1$ can be uniformly approximated, on compact subsets of $\bar{G} \backslash\{1\}$, by the weighted normalized partial sums $e^{-n z} s_{n}(n z)$. Although Proposition 3.1 above treats a very special function, it implies, in fact, the following result.

Theorem 3.2. Let $f(z)$ be analytic in $G$ and continuous on compact subsets of $\bar{G} \backslash\{1\}$. Then, given any compact subset $E$ of $\bar{G} \backslash\{1\}$, there exists a sequence of polynomials $\left\{P_{n}(z)\right\}_{n=0}^{\infty}$, with $\operatorname{deg} P_{n} \leq n$, such that

$$
\lim _{n \rightarrow \infty}\left\|e^{-n z} P_{n}(z)-f(z)\right\|_{E}=0 .
$$

Furthermore, if $f(z)$ is analytic in $G$ and continuous on $\bar{G}$ with $f(1)=0$, then it is uniformly approximable by weighted polynomials on $\bar{G}$. Thus, a sequence $\left\{P_{n}(z)\right\}_{n=0}^{\infty}$ can be chosen in this case, such that

$$
\lim _{n \rightarrow \infty}\left\|e^{-n z} P_{n}(z)-f(z)\right\| \bar{G}=0 .
$$

As we believe that the condition $f(1)=0$ is essential in Theorem 3.2 for uniform approximation on $\bar{G}$, we make the following

Conjecture. There exists a function $f$, which is analytic in $G$ and continuous on $\bar{G}$ with $f(1) \neq 0$, such that $f$ cannot be uniformly approximated on $\bar{G}$ by the weighted polynomials $\left\{e^{-n z} P_{n}(z)\right\}_{n=0}^{\infty}$, for any sequence of polynomials $\left\{P_{n}(z)\right\}_{n=0}^{\infty}$.

On choosing $f(z)$ in (3.2) to be a polynomial, it is then clear from Theorem 3.2 that any polynomial is itself approximable by weighted polynomials, locally uniformly in $\bar{G} \backslash\{1\}$. Since any function, by Mergelyan's theorem (see Gaier [5, p. 97] or Walsh [14, p. 367]), which is analytic interior to a given compact set $E$ and continuous on $E$, is uniformly approximable by polynomials on $E$ if $E$ has a connected complement, we immediately obtain: 
Corollary 3.3. Let $E \subset \bar{G} \backslash\{1\}$ be a compact set with a connected complement $\overline{\mathbb{C}} \backslash E$. If $f$ is analytic in the interior of $E$ and continuous on $E$, then there exists a sequence of polynomials $\left\{P_{n}(z)\right\}_{n=0}^{\infty}$ such that $f$ is uniformly approximable on $E$ by the weighted polynomials $\left\{e^{-n z} P_{n}(z)\right\}_{n=0}^{\infty}$.

In fact, it is possible to even give the rates of convergence of such weighted polynomials to an analytic function. We state below examples of such results, which are analogues of the classical Bernstein-Walsh overconvergence theorems (cf. [14, pp. 75-78]). For each $r$ with $0<r \leq 1$, the set

$$
S_{r}:=\{z \in \mathbb{C}:|\varphi(z)|=r, \quad|z| \leq 1, \quad 0<r \leq 1\}
$$

is an associated level curve of the mapping $\varphi$. Clearly, $S_{r} \subset G$ for any $r$ with $0<r<1$, and $S_{1}=S$. We also introduce the notation $G_{r}:=\operatorname{Int} S_{r}$, where, by definition, $G_{1}=G$.

Theorem 3.4. Let $(r, R)$ be a pair of numbers with $0<r<R \leq 1$. Then, a function $f$ is analytic in $G_{R}$ if and only if there exists a sequence of polynomials $\left\{P_{n}(z)\right\}_{n=0}^{\infty}$, with $\operatorname{deg} P_{n} \leq n$, such that

$$
\limsup _{n \rightarrow \infty}\left\|e^{-n z} P_{n}(z)-f(z)\right\|_{S_{r}}^{1 / n} \leq \frac{r}{R} .
$$

Denoting the collection of all complex polynomials of degree at most $n$ by $\pi_{n}$, we continue with

Corollary 3.5. Let

$$
E_{n}^{W}\left(f, \bar{G}_{r}\right):=\inf _{P_{n} \in \pi_{n}}\left\|e^{-n z} P_{n}(z)-f(z)\right\|_{S_{r}}
$$

be the error of the best weighted approximation on $S_{r}$ (or, equivalently, $\bar{G}_{r}$ ) for a function $f$ analytic in $G_{R}$, where $0<r<R<1$. Then, the function $f$ has a singularity on $S_{R}$ if and only if

$$
\limsup _{n \rightarrow \infty}\left[E_{n}^{W}\left(f, \bar{G}_{r}\right)\right]^{1 / n}=\frac{r}{R} .
$$

An easy but important idea, related to weighted approximation with an exponential weight, is the shift invariant property.

Proposition 3.6. Suppose that an arbitrary function, analytic in the interior of compact set $E$ and continuous on $E$, is uniformly approximable by the weighted polynomials $\left\{e^{-n z} P_{n}(z)\right\}_{n=0}^{\infty}$. Then, the same is true for any translation $\{\zeta+E\}$ of the set $E$, where $\zeta \in \mathbb{C}$.

Theorem 3.7. If a domain $H$, or any of its translations, contains $\bar{G}$, then any analytic function in $H$ (not identically zero) cannot be approximated, locally uniformly in $H$, by weighted polynomials.

Theorem 3.7 and Proposition 3.6 show that $G$ is the largest universal domain in the complex plane for weighted approximation of analytic functions, where its natural shape is inherited from the weighted normalized partial sums $e^{-n z} s_{n}(n z)$. However, one may consider weighted approximation of analytic functions in domains of different shape; e.g., disks seem to be reasonable candidates. Certainly, any disk contained in $G$ has the desired weighted approximation property, but we shall prove that the largest disk with the weighted approximation property has radius $\frac{1}{2}$, which is larger than any disk contained in $G$. 
Theorem 3.8. Any function, analytic in an open disk of radius $\frac{1}{2}$, can be approximated by weighted polynomials, $\left\{e^{-n z} P_{n}(z)\right\}_{n=0}^{\infty}$, uniformly on compact subsets of the disk. Furthermore, if $H$ is a domain containing a closed disk of radius $\frac{1}{2}$, then no analytic (not identically zero) function in $H$ can be approximated by such weighted polynomials, locally uniformly in $H$.

With the specific pair

$$
(G, W(z))
$$

where $G$ is the Jordan domain in $\mathbb{C}$ determined by the Szegö curve $S$ (cf. (1.3)), and where $W(z):=e^{-z}$ is the weight function, it is shown here in Theorem 3.2 that any function, analytic in $G$ and continuous on compact subsets of $\bar{G} \backslash\{1\}$, is uniformly approximable, on compact subsets of $\bar{G} \backslash\{1\}$, by weighted polynomials $\left\{e^{-n z} P_{n}(z)\right\}_{n=0}^{\infty}$, where $\operatorname{deg} P_{n} \leq n$. More generally, let $\mathcal{G}$ be an arbitrary Jordan domain in $\mathbb{C}$, and let $W(z)$ be an arbitrary weight function, which is analytic in $\mathcal{G}$ and continuous on $\overline{\mathcal{G}}$, with $W(z) \neq 0$ on $\overline{\mathcal{G}}$. In a subsequent paper, we characterize the pairs

$$
(\mathcal{G}, W(z))
$$

which similarly have the property that, given any compact subset $E \subset \mathcal{G}$ and any function $f(z)$ which is analytic in $\mathcal{G}$, there exists a sequence of weighted polynomials $\left\{W^{n}(z) P_{n}(z)\right\}_{n=0}^{\infty}$, with $\operatorname{deg} P_{n} \leq n$, such that

$$
\lim _{n \rightarrow \infty}\left\|f-W^{n} P_{n}\right\|_{E}=0
$$

and we also give applications of this characterization.

\section{Weighted Potentials}

In this section, we discuss some weighted potential problems which provide important tools in the derivation of our results.

Let $E \subset \mathbb{C}$ be an arbitrary compact set of positive logarithmic capacity (cf. Landkof [7, p. 167]). Consider the specific weight function

$$
w(z):=\left|e^{-z}\right|=e^{-\operatorname{Re} z}, \quad z \in \mathbb{C} .
$$

Note that $w(z)$ is continuous on any compact subset of $\mathbb{C}$. We set $Q(z):=\operatorname{Re} z$, so that

$$
w(z)=e^{-Q(z)}, \quad z \in \mathbb{C} .
$$

Let $\mathcal{M}(E)$ denote the class of all positive Borel measures $\mu$ on $\mathbb{C}$ which are supported on $E$ and have total mass unity, i.e., $\mu(\mathbb{C})=1$. We consider the following weighted energy problem:

For the weighted energy integral

$$
I_{E}(\mu):=\iint \log \frac{1}{|z-t| w(z) w(t)} d \mu(z) d \mu(t), \quad \mu \in \mathcal{M}(E),
$$

find

$$
V_{E}:=\inf _{\mu \in \mathcal{M}(E)} I_{E}(\mu)
$$

and identify the extremal measure $\mu_{E} \in \mathcal{M}(E)$ for which the infimum in (4.4) is attained.

We remark that $\left.w\right|_{E}$ (i.e., the restriction of $w$ to $E$ ) is an admissible weight, in the sense of Mhaskar and Saff [10] and Saff and Totik [11], and therefore, we can 
use the general weighted potential theory developed there to explicitly solve the problem above and to find the extremal measures for certain sets.

Recalling the notation $G_{r}:=\{z \in \mathbb{C}:|\varphi(z)|<r,|z| \leq 1\}, 0<r \leq 1$, let $\omega\left(0, \cdot, G_{r}\right)$ be the harmonic measure at $z=0$ with respect to the domain $G_{r}$. This harmonic measure can be characterized in a way similar to that of (2.1), i.e.,

$$
\omega\left(0, B, G_{r}\right)=m_{r}\left(\varphi\left(B \cap S_{r}\right)\right)
$$

where $d m_{r}:=d \theta /(2 \pi)$ on $|w|=r, 0<r \leq 1$, for any Borel set $B \subset \mathbb{C}$. The logarithmic potential of a Borel measure $\mu$, with compact support, is defined, as usual, by

$$
U^{\mu}(z):=\int \log \frac{1}{|z-t|} d \mu(t)
$$

Theorem 4.1. The solution for the weighted energy problem in (4.4) for the weight function $w(z)$ of (4.1) on $\bar{G}_{r}, 0<r \leq 1$, is given by

$$
\mu_{\bar{G}_{r}}:=\omega\left(0, \cdot, G_{r}\right) .
$$

Furthermore,

$$
U^{\mu_{\bar{G}_{r}}}(z)+Q(z)= \begin{cases}1-\log r, & z \in \bar{G}_{r}, \\ 1-\log |\varphi(z)|, & z \in \mathbb{C} \backslash G_{r},\end{cases}
$$

where $\varphi(z):=z e^{1-z}$ and $Q(z):=\operatorname{Re} z$.

Proof. Let $\delta_{0}$ be the unit point mass at $z=0$. It is known that the balayage of $\delta_{0}$ to $\partial G_{r}=S_{r}$, denoted by $\hat{\delta}_{0}$, exists and $\hat{\delta}_{0}=\omega\left(0, \cdot, G_{r}\right)$ (cf. [7, p. 222]). Since $G_{r}$ is regular with respect to the Dirichlet problem, then, by the properties of balayage (cf. Theorem II.4.1 (a) of [11] or [7, p. 209]) and by the definition in (4.6),

$$
U^{\omega\left(0, \cdot, G_{r}\right)}(z)=U^{\delta_{0}}(z)=\log \frac{1}{|z|},
$$

where $z \in \mathbb{C} \backslash G_{r}$. From (4.9), it follows that $U^{\omega\left(0, \cdot, G_{r}\right)}(z)$ is continuous on $S_{r}=$ supp $\omega\left(0, \cdot, G_{r}\right)$ and, therefore, is continuous in $\mathbb{C}$ by Theorem II.3.5 of [11] (see also Theorem 1.7 of $\left[7\right.$, p. 69]). Assume that $z \in S_{r}$, so that, from (3.4), $\log \frac{1}{|z|}+\operatorname{Re} z=$ $1-\log r$. Then, with (4.9), this gives

$$
U^{\omega\left(0, \cdot, G_{r}\right)}(z)+Q(z)=\log \frac{1}{|z|}+\operatorname{Re} z=1-\log r .
$$

Observe from the definitions above that $U^{\omega\left(0, \cdot, G_{r}\right)}(z)+Q(z)$ is harmonic in $G_{r}$ and is identically constant on the boundary $S_{r}$. By the maximum-minimum principle for harmonic functions, this implies that

$$
U^{\omega\left(0, \cdot, G_{r}\right)}(z)+Q(z) \equiv 1-\log r, \quad z \in \bar{G}_{r} .
$$

It follows from Theorem I.3.3 of [11] and (4.10) that the solution of the weighted energy problem (4.4) for the weight (4.1) on $\bar{G}_{r}$ is given by

$$
\mu_{\bar{G}_{r}}=\omega\left(0, \cdot, G_{r}\right),
$$

which is the desired result of (4.7). Also, the above relation, when used in (4.10), gives the first desired result of (4.8). We note that the constant

$$
F_{\bar{G}_{r}}:=1-\log r
$$


is the modified Robin constant (see Theorem I.1.3 of [11] or [10, p. 109]), which is connected to the solution of the energy problem by

$$
F_{\bar{G}_{r}}=V_{\bar{G}_{r}}-\int Q(t) d \mu_{\bar{G}_{r}}(t)
$$

Finally, the last equality of (4.8) of Theorem 4.1 follows from (4.9) and the definitions of $\varphi(z)$ and $Q(z)$.

Corollary 4.2. For any polynomial $P_{n}(z)$ with $\operatorname{deg} P_{n} \leq n$, we have

$$
\left|e^{-n z} P_{n}(z)\right| \leq\left\|e^{-n z} P_{n}(z)\right\|_{S_{r}}\left(\frac{|\varphi(z)|}{r}\right)^{n},
$$

where $z \in \mathbb{C} \backslash G_{r}, n \geq 0$ and $0<r \leq 1$.

We remark that (4.12) is the weighted analogue of the Bernstein-Walsh lemma for polynomials (cf. [14, p. 77]).

Proof. Since $\left|e^{-n z} P_{n}(z)\right| \leq\left\|e^{-n z} P_{n}(z)\right\|_{S_{r}}$ for any $z \in \bar{G}_{r}$, it follows from Theorem III.2.1 of [11] (see also Theorem 4.1 of [10]) and Theorem 4.1 that

$$
\left|P_{n}(z)\right| \leq\left\|e^{-n z} P_{n}(z)\right\|_{S_{r}} \exp \left\{n\left(F_{\bar{G}_{r}}-U^{\mu_{\bar{G}_{r}}}(z)\right)\right\}, \quad z \in \mathbb{C} .
$$

Multiplying (4.13) by the $n$-th power of the weight $w(z)=e^{-Q(z)}$, and taking into account (4.11) and the second part of (4.8), proves the corollary.

In the rest of this section, we give the solution of the weighted energy problem for the weight $w(z)$ of (4.1) on a family of disks $D_{r}:=\{z:|z|<r\}$. For additional notation, we say that a property holds quasi-everywhere (written q.e.) if it holds everywhere, with the exception of a set of zero logarithmic capacity.

Theorem 4.3. The extremal measure $\mu_{\bar{D}_{r}}$ for the weight (4.1) on $\bar{D}_{r}$, where $0<$ $r \leq \frac{1}{2}$, satisfies

$$
\operatorname{supp} \mu_{\bar{D}_{r}}=\partial D_{r}=\{z:|z|=r\}
$$

and is absolutely continuous with respect to angular measure $d \theta$ on $\partial D_{r}$, with

$$
d \mu_{\bar{D}_{r}}(\theta)=\frac{1}{2 \pi}(1-2 r \cos \theta) d \theta \text { on }|z|=r .
$$

Furthermore, if $0<r \leq \frac{1}{2}$, then

$$
U^{\mu \bar{D}_{r}}(z)+Q(z)=\left\{\begin{array}{l}
\log \frac{1}{r}, z \in \bar{D}_{r}, \\
\log \frac{1}{|z|}+\left(1-\frac{r^{2}}{|z|^{2}}\right) \operatorname{Re} z, z \in \mathbb{C} \backslash D_{r} .
\end{array}\right.
$$

However, if $r>\frac{1}{2}$, then $\operatorname{supp} \mu_{\bar{D}_{r}} \subset \partial D_{r}$ with $\operatorname{supp} \mu_{\bar{D}_{r}} \neq \partial D_{r}$, and the following is satisfied:

$$
U^{\mu_{\bar{D}_{r}}}(z)+Q(z)=F_{\bar{D}_{r}}, \quad \text { q.e. on } \operatorname{supp} \mu_{\bar{D}_{r}},
$$

and

$$
U^{\mu_{\bar{D}_{r}}}(z)+Q(z)>F_{\bar{D}_{r}}, \quad z \in D_{r}
$$

where $F_{\bar{D}_{r}}$ is the modified Robin constant for $\bar{D}_{r}$. 
Proof. Let $\mu_{\bar{D}_{r}}$ be the extremal measure for (4.4) on $\bar{D}_{r}$, with $r>0$. Since $Q(z)$ is harmonic in $D_{r}$, then $\operatorname{supp} \mu_{\bar{D}_{r}} \subset \partial D_{r}$ by Theorem IV.1.10(a) of [11]. It is also known from Theorem I.1.3 of [11] (or Theorem 3.1 of [10]) that

$$
U^{\mu_{\bar{D}_{r}}}(z)+Q(z) \geq F_{\bar{D}_{r}}
$$

holds for quasi-every $z \in \bar{D}_{r}$, and

$$
U^{\mu \bar{D}_{r}}(z)+Q(z)=F_{\bar{D}_{r}}
$$

holds quasi-everywhere (q.e.) on $\operatorname{supp} \mu_{\bar{D}_{r}}$, where $F_{\bar{D}_{r}}$ is the modified Robin constant for $\bar{D}_{r}$.

Suppose that $\operatorname{supp} \mu_{\bar{D}_{r}}=\partial D_{r}$. Observe that the function $U^{\mu_{\bar{D}_{r}}}(z)+Q(z)$ is harmonic in $D_{r}$ and is continuous in $\mathbb{C}$ by Theorem I.4.8 (ii) of [11]. Therefore, from (4.20) and the uniqueness theorem for harmonic functions, it follows that

$$
U^{\mu \bar{D}_{r}}(z)+Q(z) \equiv F_{\bar{D}_{r}}, \quad z \in \bar{D}_{r} .
$$

However, from (4.6) and the definition of $Q(z)$, we have that

$$
U^{\mu_{\bar{D}_{r}}(0)}+Q(0)=\int \log \frac{1}{|t|} d \mu_{\bar{D}_{r}}(t)=\log \frac{1}{r},
$$

because $\operatorname{supp} \mu_{\bar{D}_{r}}=\partial D_{r}$ and because $\mu_{\bar{D}_{r}}(\mathbb{C})=1$. Thus, we see from (4.21) that

$$
F_{\bar{D}_{r}}=\log \frac{1}{r} \text {. }
$$

Next, it is easily seen that $U^{\mu_{\bar{D}_{r}}}(z)+\log \frac{|z|}{r}$ is a harmonic function in $\mathbb{C} \backslash \bar{D}_{r}$, with boundary values satisfying

$$
U^{\mu \bar{D}_{r}}(z)+\log \frac{|z|}{r}=\log \frac{1}{r}-Q(z), \quad|z|=r,
$$

by (4.21) and (4.22). Thus, on solving the above exterior Dirichlet problem, we find that

or equivalently,

$$
U^{\mu \bar{D}_{r}}(z)+\log \frac{|z|}{r}=\log \frac{1}{r}-r^{2} \operatorname{Re} \frac{1}{z}, \quad|z| \geq r,
$$

$$
U^{\mu_{\bar{D} r}}(z)=\log \frac{1}{|z|}-r^{2} \operatorname{Re} \frac{1}{z}, \quad|z| \geq r,
$$

while for the associated interior Dirichlet problem, we have from (4.21) and (4.22) that

$$
U^{\mu_{\bar{D}_{r}}}(z)=\log \frac{1}{r}-\operatorname{Re} z, \quad|z| \leq r .
$$

Using our knowledge of the logarithmic potential of an extremal measure, we can recover the measure itself (see Theorem IV.2.3 of [11]) by means of

$$
d \mu_{\bar{D}_{r}}(\theta)=-\frac{1}{2 \pi}\left(\frac{\partial U^{\mu_{\bar{D}_{r}}}}{\partial n_{+}}(\theta)+\frac{\partial U^{\mu_{\bar{D}_{r}}}}{\partial n_{-}}(\theta)\right) r d \theta,
$$

where $d \theta$ is the angular measure on $|z|=r$, and where $n_{+}$and $n_{-}$are respectively the inner and the outer normals to the circle $|z|=r$. A direct calculation using (4.24) and (4.25) gives

$$
d \mu_{\bar{D}_{r}}(\theta)=-\frac{1}{2 \pi}\left(\operatorname{Re} e^{i \theta}+\left(-\frac{1}{r}+\operatorname{Re} e^{-i \theta}\right)\right) r d \theta,
$$


which reduces to

$$
d \mu_{\bar{D}_{r}}(\theta)=\frac{1}{2 \pi}(1-2 r \cos \theta) d \theta .
$$

Thus, (4.15) of Theorem 4.3 follows from (4.26), and (4.16) of Theorem 4.3 follows from (4.21), (4.22), and (4.24). Note that if $0<r \leq \frac{1}{2}$, then (4.26) is the density function of the unit Borel measure satisfying condition (4.21), and this implies that we indeed have found the extremal measure by Theorem I.3.3 of [11]. However if $r>\frac{1}{2}$, then the density function in (4.26) is clearly negative in a neighborhood of $\theta=0$, i.e., the measure of (4.26) cannot be a Borel (positive) measure. This contradiction forces us to accept that $\operatorname{supp} \mu_{\bar{D}_{r}}$ is a proper subset of $|z|=r$ for any $r>\frac{1}{2}$, as stated in Theorem 4.3. Equality in (4.17) follows from (4.20).

Finally, to prove (4.18), we note that $U^{\mu \bar{D}_{r}}(z)+Q(z)$ is harmonic in $\mathbb{C} \backslash \operatorname{supp} \mu_{\bar{D}_{r}}$ and continuous q.e. in $\mathbb{C}$ by Theorem I.4.4 of [11]. Suppose, contrary to (4.18), that

$$
U^{\mu \bar{D}_{r}}\left(z_{0}\right)+Q\left(z_{0}\right)=F_{\bar{D}_{r}}
$$

for some $z_{0} \in D_{r}$. Then, by the minimum principle for harmonic functions and (4.19),

$$
U^{\mu \bar{D}_{r}}(z)+Q(z)=F_{\bar{D}_{r}}
$$

for any $z \in \bar{D}_{r}$. Thus, by the uniqueness theorem,

$$
U^{\mu_{\bar{D}_{r}}}(z)+Q(z)=F_{\bar{D}_{r}}, \quad z \in \mathbb{C} \backslash \operatorname{supp} \mu_{\bar{D}_{r}},
$$

which, from (4.24) with $z=R$ (where $R \geq r$ ), would imply that

$$
-\log R-\frac{r^{2}}{R}+R=F_{\bar{D}_{r}} \text { for all } R \geq r
$$

which is clearly impossible for $R$ sufficiently large. Thus, the inequality of (4.18) follows.

The weighted analogue of the Bernstein-Walsh lemma in the case of $\bar{D}_{r}$, with $0<r \leq \frac{1}{2}$, is given in the following:

Corollary 4.4. Let $P_{n}(z)$ be a polynomial of degree at most $n, n \geq 0$. Then,

$$
\left|e^{-n z} P_{n}(z)\right| \leq\left\|e^{-n z} P_{n}(z)\right\|_{\bar{D}_{r}} \exp \left\{n\left(\log \frac{|z|}{r}+\left(\frac{r^{2}}{|z|^{2}}-1\right) \operatorname{Re} z\right)\right\},
$$

for any $z \in \mathbb{C} \backslash D_{r}$, provided that $0<r \leq \frac{1}{2}$.

If $r>\frac{1}{2}$ and if for some constant $C>0$ we have $\left\|e^{-n z} P_{n}(z)\right\|_{\bar{D}_{r}} \leq C$ for all $n \geq 0$, then

$$
\left|e^{-n z} P_{n}(z)\right| \rightarrow 0 \quad \text { as } n \rightarrow \infty
$$

locally uniformly in $D_{r}$.

Proof. We proceed in exactly the same way as in the proof of Corollary 4.2 to obtain, by Theorem III.2.1 of [11] (or Theorem 4.1 of [10]), that

$$
\left|e^{-n z} P_{n}(z)\right| \leq\left\|e^{-n z} P_{n}(z)\right\|_{\bar{D}_{r}} \exp \left\{n\left(F_{\bar{D}_{r}}-U^{\mu \bar{D}_{r}}(z)-Q(z)\right)\right\}, \quad z \in \mathbb{C} .
$$

Thus, with the above expression, (4.27) follows from (4.16) and (4.22), and (4.28) follows from (4.18). 


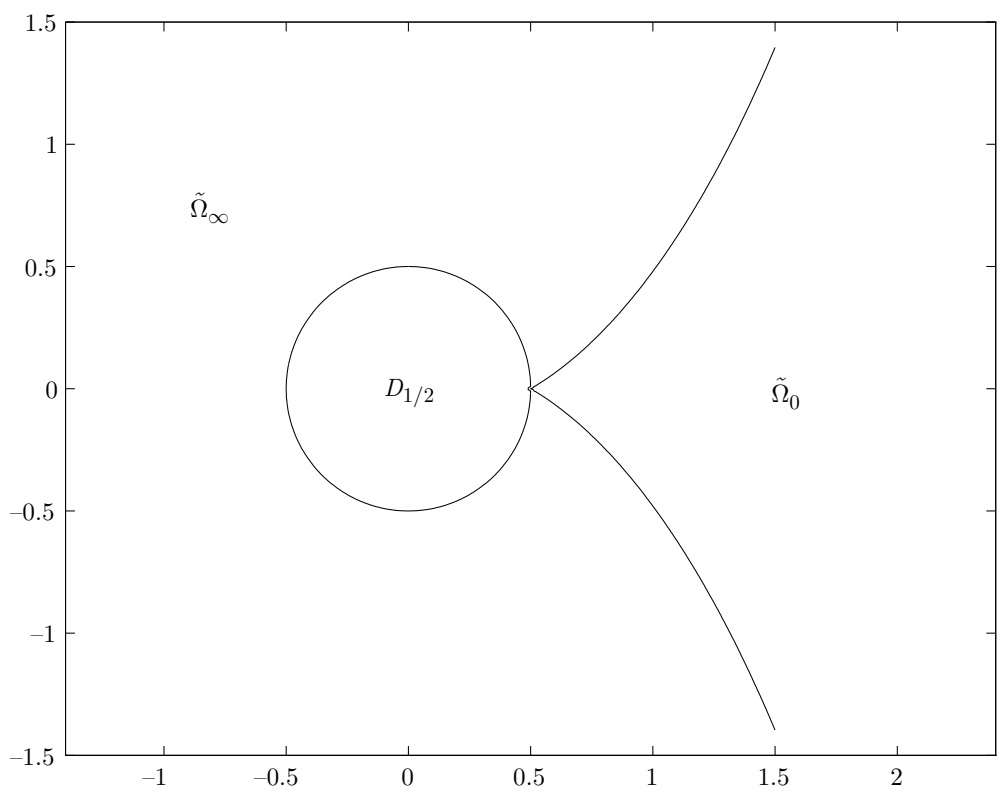

Figure 2. The extremal disk $D_{1 / 2}$ and the associated domains

In analogy with Figure 1, we show, in Figure 2, the largest disk (with radius $r=1 / 2$ from Theorem 3.8) and its associated unbounded domains $\tilde{\Omega}_{0}$ and $\tilde{\Omega}_{\infty}$. The boundaries for these domains are formed by the curve which is defined, from the right side of (4.27) for the case $r=1 / 2$, by

$$
\left\{z \in \mathbb{C}: \log \frac{|z|}{r}+\left(\frac{r^{2}}{|z|^{2}}-1\right) \operatorname{Re} z=0 \text { and }|z| \geq r\right\} .
$$

\section{Proofs}

Proof of Theorem 2.1. Consider the normalized partial sum

$$
s_{n}(n z)=\sum_{k=0}^{n} \frac{n^{k}}{k !} z^{k}=a_{n} z^{n}+\ldots, \quad n \geq 1,
$$

where the leading coefficient of $s_{n}(n z)$ is given by

$$
a_{n}=\frac{n^{n}}{n !} .
$$

Then from Stirling's formula (1.2),

$$
\lim _{n \rightarrow \infty} a_{n}^{1 / n}=e .
$$

Next, since any point $z$ of $\bar{G}$ satisfies $\left|z e^{1-z}\right| \leq 1$ and $|z| \leq 1$, it readily follows from (1.1) and (1.2) that

$$
\left|e^{-n z} s_{n}(n z)\right| \leq 1+\sqrt{\frac{n}{2 \pi}}, \quad z \in \bar{G}, n \geq 1 .
$$


Thus, for the monic normalized partial sums $s_{n}(n z) / a_{n}$, we have

$$
\limsup _{n \rightarrow \infty}\left\|e^{-n z} s_{n}(n z) / a_{n}\right\|_{\frac{G}{G}}^{1 / n} \leq \limsup _{n \rightarrow \infty}\left\{\frac{1+\sqrt{\frac{n}{2 \pi}}}{a_{n}}\right\}^{1 / n}=\frac{1}{e} .
$$

But, as $\partial G=S$ is a subset of $\bar{G}$, the above gives

$$
\limsup _{n \rightarrow \infty}\left\|e^{-n z} s_{n}(n z) / a_{n}\right\|_{S}^{1 / n} \leq \frac{1}{e} .
$$

On the other hand, for any monic polynomial $P_{n}(z)$ of degree at most $n$, we have by Theorem I.3.6 of [11] (or Corollary 4.5 of [10]) that $\left\|e^{-n z} P_{n}(z)\right\|_{S} \geq e^{-n F_{\bar{G}}}$, so that, as the case $r=1$ of (4.11) implies that $F_{\bar{G}}=1$,

$$
\liminf _{n \rightarrow \infty}\left\|e^{-n z} P_{n}(z)\right\|_{S}^{1 / n} \geq e^{-F_{\bar{G}}}=\frac{1}{e} .
$$

The inequalities (5.4) and (5.5) together then give

$$
\lim _{n \rightarrow \infty}\left\|e^{-n z} s_{n}(n z) / a_{n}\right\|_{S}^{1 / n}=\frac{1}{e}=e^{-F_{\bar{G}}},
$$

i.e., $s_{n}(n z) / a_{n}, n \geq 0$, is an asymptotically extremal monic polynomial in the terminology of [11].

Next, given any compact set $A \subset G$, it is a consequence of (1.1) that $A$ contains no zeros of $s_{n}(n z)$, provided that $n$ is sufficiently large. (More precisely, Buckholtz [2] has shown that all zeros of $s_{n}(n z)$ lie outside of $S$ for any $n \geq 1$.) Thus, from $(2.2)$,

$$
\lim _{n \rightarrow \infty} \nu_{n}\left(s_{n}(n z)\right)(A)=0,
$$

and by (5.6), Theorem 2.3(b) of [9], and Theorem 4.1, we obtain that

$$
\nu_{n}\left(s_{n}(n z)\right)(G) \stackrel{*}{\rightarrow} \omega(0, \cdot, G), \quad \text { as } n \rightarrow \infty,
$$

the desired result of (2.3) of Theorem 2.1.

Since all accumulation points of the zeros of $\left\{s_{n}(n z)\right\}_{n=1}^{\infty}$ lie on $S$, we have for any $z \in \mathbb{C} \backslash \bar{G}$ that

$$
\begin{aligned}
& \lim _{n \rightarrow \infty}\left|e^{-n z} s_{n}(n z)\right|^{1 / n}=\lim _{n \rightarrow \infty} a_{n}^{1 / n} e^{-Q(z)}\left|s_{n}(n z) / a_{n}\right|^{1 / n} \\
& =e^{1-Q(z)} \lim _{n \rightarrow \infty} \exp \left\{-U^{\nu_{n}\left(s_{n}(n z) / a_{n}\right)}(z)\right\} \\
& =\exp \{1-Q(z)\} \exp \left\{-U^{\omega(0, \cdot, G)}(z)\right\} \\
& =\exp \left\{1-Q(z)-U^{\omega(0, \cdot, G)}(z)\right\},
\end{aligned}
$$

where we successively used (5.3), (4.2), (4.6), and (2.3). We note, from the definition of the logarithmic potential in (4.6), that this above limit holds uniformly on compact subsets of $\mathbb{C} \backslash \bar{G}$. Then with $r=1$ in both (4.7) and the second expression in (4.8), the above display reduces to $|\varphi(z)|$, i.e.,

$$
\lim _{n \rightarrow \infty}\left|e^{-n z} s_{n}(n z)\right|^{1 / n}=|\varphi(z)|, \quad z \in \mathbb{C} \backslash \bar{G},
$$

uniformly on any compact subset of $\mathbb{C} \backslash \bar{G}$, which gives the final desired result of (2.4) of Theorem 2.1. 
Proof of Proposition 3.1. Let $z \in \bar{G} \backslash\{1\}$. Following Szegő [12], we make substitution $w=\zeta e^{1-\zeta}$ in (1.1), which gives

$$
1-e^{-n z} s_{n}(n z)=\frac{\sqrt{n}}{\tau_{n} \sqrt{2 \pi}} \int_{0}^{z e^{1-z}} w^{n-1} \frac{\zeta(w)}{1-\zeta(w)} d w .
$$

Our goal is to bound the modulus of the right side of (5.7) from above. Denoting the integral in (5.7) by $I$, an integration by parts and use of the relation $w(\zeta)=\zeta e^{1-\zeta}$ give us that

$$
\begin{aligned}
I & :=\int_{0}^{z e^{1-z}} w^{n-1} \frac{\zeta(w) d w}{1-\zeta(w)} \\
& =\left.\frac{w^{n}}{n} \frac{\zeta(w)}{1-\zeta(w)}\right|_{0} ^{z e^{1-z}}-\int_{0}^{z e^{1-z}} \frac{w^{n}}{n} \frac{\zeta^{\prime}(w) d w}{(1-\zeta(w))^{2}} \\
& =\frac{\left(z e^{1-z}\right)^{n}}{n} \frac{z}{1-z}-\frac{1}{n} \int_{0}^{z} \frac{\left(\zeta e^{1-\zeta}\right)^{n}}{(1-\zeta)^{2}} d \zeta \\
& =\frac{1}{n(1-z)}\left(z\left(z e^{1-z}\right)^{n}+(z-1) \int_{0}^{z} \frac{\left(\zeta e^{1-\zeta}\right)^{n}}{(1-\zeta)^{2}} d \zeta\right) .
\end{aligned}
$$

To bound $|I|$ above, assume that $z=(x+i y) \in \bar{G} \backslash\{1\}$, and choose the path of integration in the integral of $I$ to consist of the two intervals $[0, x]$ and $[x, x+i y]$. Then, as $|z| \leq 1$ and $\left|z e^{1-z}\right| \leq 1$ for all points of $\bar{G}$,

$$
\begin{aligned}
|I| & \leq \frac{1}{n|1-z|}\left\{1+|z-1|\left(\int_{0}^{|x|} \frac{d t}{(1-t)^{2}}+\int_{0}^{|y|} \frac{d s}{(1-|x|)^{2}}\right)\right\} \\
& =\frac{1}{n|1-z|}\left\{1+|z-1|\left(\frac{|x|}{1-|x|}+\frac{|y|}{(1-|x|)^{2}}\right)\right\} \\
& \leq \frac{1}{n|1-z|}\left\{1+|z-1|\left(\frac{1}{1-|x|}+\frac{|y|}{(1-|x|)^{2}}\right)\right\} .
\end{aligned}
$$

Then, it can be verified that the square with vertices \pm 1 and $\pm i$ contains $\bar{G}$. (This is shown in Figure 3.) This geometrically implies that

$$
|y| \leq 1-|x| \text { and } 1-|x| \leq|1-z| \leq \sqrt{2}(1-|x|) \text {. }
$$

Inserting these inequalities into the upper bound above for $|I|$ yields

$$
|I| \leq \frac{1}{n|1-z|}\left\{1+2 \frac{|z-1|}{1-|x|}\right\} \leq \frac{1}{n|1-z|}\{1+2 \sqrt{2}\} \leq \frac{4}{n|1-z|}
$$

for any $z=x+i y \in \bar{G}$. This bound, applied to (5.7), then gives the desired result of (3.1) of Proposition 3.1.

Proof of Theorem 3.2. First, fix a small $\delta$ with $1>\delta>0$ and consider the domain $G_{\delta}:=G \backslash\{z:|z-1| \leq \delta\}$. It follows immediately from (3.1) that

$$
\left\|e^{-n z} s_{n}(n z)-1\right\|_{\bar{G}_{\delta}} \leq \frac{4}{\sqrt{2 \pi n}}\left\|\frac{1}{z-1}\right\|_{\bar{G}_{\delta}}=\frac{4}{\sqrt{2 \pi n} \delta}, \quad n \geq 1
$$




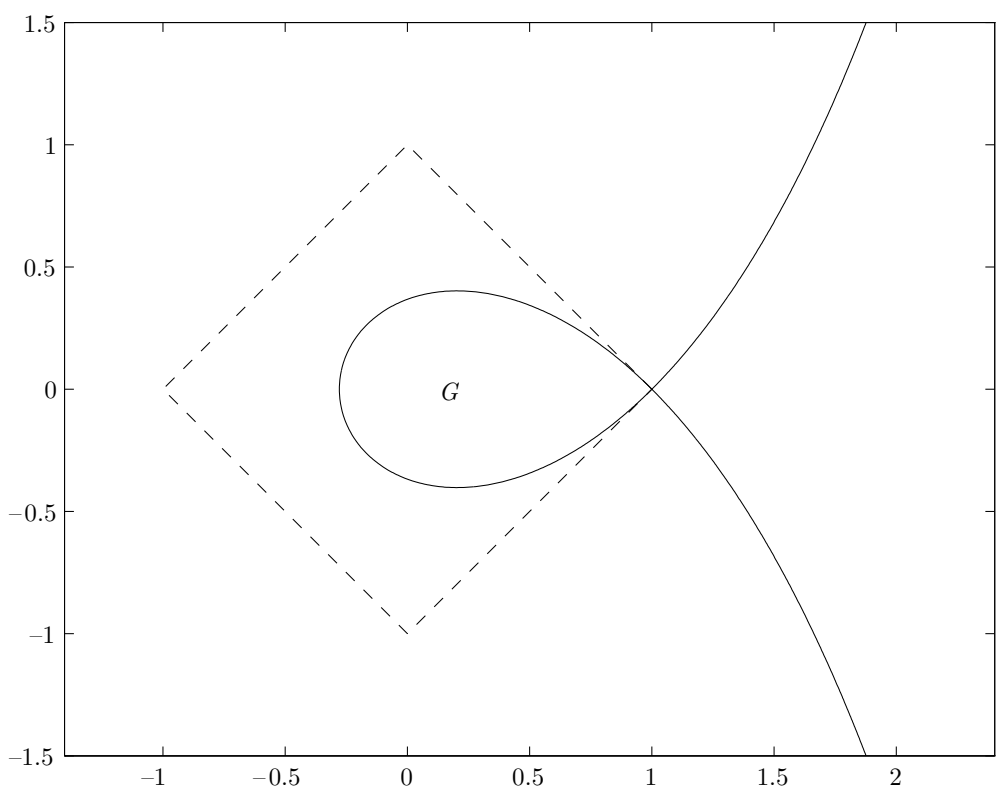

FiguRE 3. The Szegö domain $G$ and the covering square

so that

$$
\left\|e^{-n z} s_{n}(n z)-1\right\|_{\bar{G}_{\delta}} \rightarrow 0, \text { as } n \rightarrow \infty .
$$

Multiplying (3.1) by $e^{-k z}$, we similarly observe that, for any fixed $k=0,1,2, \ldots$,

$$
\left\|e^{-(n+k) z} s_{n}(n z)-e^{-k z}\right\|_{\bar{G}_{\delta}} \leq \frac{4}{\sqrt{2 \pi n} \delta}\left\|e^{-k z}\right\|_{\bar{G}_{\delta}}, \quad n \geq 1,
$$

so that

$$
\left\|e^{-(n+k) z} s_{n}(n z)-e^{-k z}\right\|_{\bar{G}_{\delta}} \rightarrow 0, \text { as } n \rightarrow \infty .
$$

This means that $e^{-k z}$, for any $k \geq 0$, can be uniformly approximated on $\bar{G}_{\delta}$ by the weighted polynomials $\left\{e^{-n z} s_{n-k}((n-k) z)\right\}_{n=k}^{\infty}$. Therefore, any complex polynomial in $e^{-z}$, say $Q_{m}\left(e^{-z}\right)=\sum_{j=0}^{m} c_{j} e^{-j z}$, can also be uniformly approximated on $\bar{G}_{\delta}$ by such weighted polynomials $e^{-n z} P_{n}(z)$. In fact, it is easy to see from (5.9) that these polynomials $P_{n}(z)$ in this case can be chosen to be

$$
P_{n}(z):=\sum_{j=0}^{m} c_{j} s_{n-j}((n-j) z), \quad n \geq m .
$$

If we show that any function $f(z)$ which is analytic in $G$ and continuous on compact subsets of $\bar{G} \backslash\{1\}$ is uniformly approximable on $\bar{G}_{\delta}$ by polynomials $Q_{m}\left(e^{-z}\right)$, then (3.2) of Theorem 3.2 will follow. Indeed, as $\partial G_{\delta}$ is a Jordan curve (with interior $G_{\delta}$ ), so is its image, $\partial H_{\delta}$ (with interior $H_{\delta}$ ) in the $t$-plane, under the conformal mapping $\Phi(z):=e^{-z}=t$. As can be readily verified, for any $\delta$ with $0 \leq \delta \leq 1$, the image of $\bar{G}_{\delta}$, under $\Phi(z)=t$, lies in the open right-half plane of the $t$-plane, and is symmetric about the positive real axis. Hence, on cutting the $t$-plane along the 
negative real axis, then $f(-\log t)$ is analytic and single-valued in $H_{\delta}$ and continuous on $\partial H_{\delta}$. Thus, by Mergelyan's Theorem (cf. [5, p. 97]) $f(-\log t)$ can be uniformly approximated on $\bar{H}_{\delta}$ by the polynomials $Q_{m}(t)$. But this means that $f(z)$ can be uniformly approximated by $Q_{m}\left(e^{-z}\right)$ on $\bar{G}_{\delta}$.

To prove the second assertion of Theorem 3.2, we note, on multiplying (3.1) by $(z-1) e^{-k z}$, that

$$
\left\|e^{-(n+k) z}\left((z-1) s_{n}(n z)\right)-(z-1) e^{-k z}\right\|_{\bar{G}} \leq \frac{4\left\|e^{-k z}\right\|_{\bar{G}}}{\sqrt{2 \pi n}},
$$

which implies that any function of the form $(z-1) e^{-k z}, k \geq 1$, can be approximated by weighted polynomials $e^{-n z} P_{n}(z)$, uniformly on $\bar{G}$. It follows, for any polynomial $Q_{m}(t)$ with $Q_{m}(0)=0$, that $(z-1) Q_{m}\left(e^{-z}\right)$ is uniformly approximable by weighted polynomials $e^{-n z} P_{n}(z)$ on $\bar{G}$. Now, assume in addition that $f(z)$ is analytic at $z=1$. On defining the function

$$
v(z):=\frac{f(z) e^{z}}{z-1}
$$

it follows, since $f(1)=0$ by hypothesis, that $v(z)$ is analytic in $G$ and continuous on $\bar{G}$. Then, by the previous argument (with $G_{\delta}$ and $H_{\delta}$ being replaced, respectively, by $G$ and $H$ ) and with the same mapping $\Phi(z):=e^{-z}=t$, it similarly follows that $v(-\log t)$ can be uniformly approximated on $\bar{H}$ by the polynomials $\tilde{Q}_{m-1}(t)$, so that

$$
\left\|\frac{f(z) e^{z}}{z-1}-\tilde{Q}_{m-1}\left(e^{-z}\right)\right\|_{\bar{G}}=\left\|v(-\log t)-\tilde{Q}_{m-1}(t)\right\|_{\bar{H}} \rightarrow 0,
$$

as $m \rightarrow \infty$. On setting $Q_{m}(t):=t \tilde{Q}_{m-1}(t)$ so that $Q_{m}(0)=0$, the above display, after multiplying through by $(z-1) e^{-z}$, gives that

$$
\left\|f(z)-(z-1) Q_{m}\left(e^{-z}\right)\right\|_{\bar{G}} \rightarrow 0, \text { as } m \rightarrow \infty,
$$

which shows that $f(z)$ is uniformly approximable on $\bar{G}$ by weighted polynomials $e^{-n z} P_{n}(z)$. To complete the proof, we now drop the hypothesis that $f(z)$ is analytic at $z=1$. Let $P_{n}(z)$ be the best uniform approximation from $\pi_{n}$ to $f(z)$ on $\bar{G}$. By Mergelyan's Theorem again,

$$
\lim _{n \rightarrow \infty}\left\|f(z)-P_{n}(z)\right\|_{\bar{G}}=0 .
$$

For each $n \geq 0$, define $\tilde{P}_{n}(z):=P_{n}(z)-P_{n}(1)$, so that $\tilde{P}_{n}(1)=0$. Because $f(1)=0$, we see that

$$
\left|P_{n}(1)\right|=\left|f(1)-P_{n}(1)\right| \leq\left\|f-P_{n}\right\|_{\bar{G}},
$$

which implies that

$$
\left\|f-\tilde{P}_{n}\right\|_{\bar{G}} \leq\left\|f-P_{n}\right\|_{\bar{G}}+\left|P_{n}(1)\right| \leq 2\left\|f-P_{n}\right\|_{\bar{G}},
$$

i.e., $f(z)$ can be uniformly approximated in $\bar{G}$ by $\tilde{P}_{n}(z)$. But as our previous proof can be applied to each $\tilde{P}_{n}(z)$, it follows that $f(z)$ can be uniformly approximated on $\bar{G}$ by weighted polynomials.

Proof of Theorem 3.4. Given the pair of numbers $(r, R)$ with $0<r<R \leq 1$, suppose that $f(z)$ is analytic in $G_{R}$. For each $n \geq 0$, let $\left\{z_{k}^{(n+1)}\right\}_{k=1}^{n+1}$ be $n+1$ points (to be specified below) such that $\left\{z_{k}^{(n+1)}\right\}_{k=1}^{n+1} \subset G_{R}$. Then, from the Hermite 
interpolation formula, the polynomial $P_{n}(z)$, which interpolates $e^{n z} f(z)$ in the $n+1$ points $\left\{z_{k}^{(n+1)}\right\}_{k=1}^{n+1}$, is given (cf. [14, p. 50]) by

$$
e^{n z} f(z)-P_{n}(z)=\frac{\omega_{n+1}(z)}{2 \pi i} \int_{S_{R-\epsilon}} \frac{f(t) e^{n t} d t}{(t-z) \omega_{n+1}(t)}
$$

where $\omega_{n+1}(z):=\prod_{k=1}^{n+1}\left(z-z_{k}^{(n+1)}\right)$ and where $z \in G_{R-\epsilon}$; here, $\epsilon>0$ is chosen sufficiently small so that $\left\{z_{k}^{(n+1)}\right\}_{k=1}^{n+1} \subset G_{R-\epsilon}$. Dividing by $e^{n z}$ in (5.10) gives

$$
f(z)-e^{-n z} P_{n}(z)=\frac{e^{-n z} \omega_{n+1}(z)}{2 \pi i} \int_{S_{R-\epsilon}} \frac{f(t) d t}{(t-z) e^{-n t} \omega_{n+1}(t)},
$$

for $z \in G_{R-\epsilon}$.

Let $\nu_{n}\left(\omega_{n}\right)$ be the normalized counting measure of the zeros of $\omega_{n}(z)$, i.e. (see $(2.2))$

$$
\nu_{n}\left(\omega_{n}\right)=\frac{1}{n} \sum_{k=1}^{n} \delta_{z_{k}^{(n)}}, \quad n \geq 1
$$

Then, from the definition in (4.6),

$$
\left|\omega_{n}(z)\right|=\exp \left\{-n U^{\nu_{n}\left(\omega_{n}\right)}(z)\right\}, \quad n \geq 1 .
$$

For each $r$ with $0<r<R$, we now choose an interpolation scheme in (5.10) which satisfies

$$
\left\{z_{k}^{(n)}\right\}_{k=1}^{n} \subset S_{r}, \quad n \geq 1
$$

and

$$
\nu_{n}\left(\omega_{n}\right) \stackrel{*}{\rightarrow} \omega\left(0, \cdot, G_{r}\right), \quad \text { as } n \rightarrow \infty .
$$

As an example of an interpolation where (5.14) and (5.15) are valid, one can take the preimages of equally spaced points on $|w|=r$ under the conformal map $w=$ $\varphi(z)=z e^{1-z}$, i.e., for $\psi:=\varphi^{[-1]}$, we define

$$
z_{k}^{(n)}:=\psi\left(r e^{i \frac{2 \pi k}{n}}\right), \quad 1 \leq k \leq n, \quad n=1,2, \ldots .
$$

It follows from (5.13)-(5.15) that

$$
\begin{aligned}
\lim _{n \rightarrow \infty}\left|\omega_{n}(z)\right|^{1 / n} & =\lim _{n \rightarrow \infty} \exp \left\{-U^{\nu_{n}\left(\omega_{n}\right)}(z)\right\} \\
& =\exp \left\{-U^{\omega\left(0, \cdot, G_{r}\right)}(z)\right\},
\end{aligned}
$$

which holds locally uniformly in $\mathbb{C} \backslash \bar{G}_{r}$. Taking any $\epsilon$ small enough so that $r+\epsilon<$ $R-\epsilon$, we estimate the difference in (5.11) by

$$
\begin{aligned}
& \left\|f(z)-e^{-n z} P_{n}(z)\right\|_{\bar{G}_{r}} \leq\left\|f(z)-e^{-n z} P_{n}(z)\right\|_{\bar{G}_{r+\epsilon}} \\
& \leq \frac{\left\|e^{-n z} \omega_{n+1}(z)\right\|_{S_{r+\epsilon}}\|f\|_{S_{R-\epsilon}}}{2 \pi \operatorname{dist}\left(S_{r+\epsilon}, S_{R-\epsilon}\right) \cdot \min _{t \in S_{R-\epsilon}}\left|e^{-n t} \omega_{n+1}(t)\right|} .
\end{aligned}
$$

Thus, we obtain, by (5.17), (4.2) and (4.8), that

$$
\limsup _{n \rightarrow \infty}\left\|f(z)-e^{-n z} P_{n}(z)\right\|_{S_{r}}^{1 / n} \leq \frac{e^{\log (r+\epsilon)-1}}{e^{\log (R-\epsilon)-1}}=\frac{r+\epsilon}{R-\epsilon} .
$$


Letting $\epsilon \rightarrow 0$, we have established (3.5) of Theorem 3.4.

To show that the converse part of Theorem 3.4 is valid, suppose that (3.5) holds true for $r$ with $0<r<R \leq 1$. Then, the rest of the proof is a classical converse theorem argument (see [14, p. 81], for example). By the uniform convergence on $\bar{G}_{r}$, the function $f(z)$ can be represented, in a telescopic series, as

$$
f(z)=e^{-n z} P_{n}(z)+\sum_{k=n}^{\infty}\left(e^{-(k+1) z} P_{k+1}(z)-e^{-k z} P_{k}(z)\right), \quad z \in \bar{G}_{r} .
$$

Thus,

$$
\left|f(z)-e^{-n z} P_{n}(z)\right| \leq \sum_{k=n}^{\infty}\left|e^{-(k+1) z} P_{k+1}(z)-e^{-k z} P_{k}(z)\right| .
$$

For any $\epsilon>0$, we have from (3.5) that

$$
\left\|f(z)-e^{-k z} P_{k}(z)\right\|_{\bar{G}_{r}} \leq\left(\frac{r}{R-\epsilon}\right)^{k},
$$

if $k \geq n$ is sufficiently large. This gives us

$$
\begin{aligned}
& \left\|e^{-(k+1) z} P_{k+1}(z)-e^{-k z} P_{k}(z)\right\|_{\bar{G}_{r}} \\
& \leq\left\|f(z)-e^{-k z} P_{k}(z)\right\|_{\bar{G}_{r}}+\left\|f(z)-e^{-(k+1) z} P_{k+1}(z)\right\|_{\bar{G}_{r}} \\
& \leq C_{1}\left(\frac{r}{R-\epsilon}\right)^{k}, \quad k \geq n,
\end{aligned}
$$

where $C_{1}$ is a constant, independent of $k$. Using Corollary 4.2, we obtain from the above estimate that

$$
\begin{aligned}
& \left|e^{-(k+1) z} P_{k+1}(z)-e^{-k z} P_{k}(z)\right| \leq C_{1}\left(\frac{r}{R-\epsilon}\right)^{k}\left(\frac{|\varphi(z)|}{r}\right)^{k} \\
& =C_{1}\left(\frac{|\varphi(z)|}{R-\epsilon}\right)^{k}, \quad k \geq n .
\end{aligned}
$$

If $|\varphi(z)|=R-2 \epsilon$, i.e., $z \in S_{R-2 \epsilon}$, then the telescopic series (5.18) converges to the analytic continuation of $f(z)$ in $G_{R-2 \epsilon}$. Thus, from (5.19) and the above inequalities,

$$
\left\|f(z)-e^{-n z} P_{n}(z)\right\|_{\bar{G}_{R-2 \epsilon}} \leq C_{2}\left(\frac{R-2 \epsilon}{R-\epsilon}\right)^{n},
$$

for any sufficiently large $n$. Hence, the sequence $\left\{e^{-n z} P_{n}(z)\right\}_{n=0}^{\infty}$ converges to the analytic continuation of $f(z)$, uniformly on $\bar{G}_{R-2 \epsilon}$. Since $\epsilon>0$ can be taken arbitrarily small, then $f(z)$ must be analytic in $G_{R}$.

Proof of Corollary 3.5. If $f(z)$ is analytic in $G_{R}$, then by Theorem 3.4,

$$
\limsup _{n \rightarrow \infty}\left[E_{n}^{W}\left(f, \bar{G}_{r}\right)\right]^{1 / n} \leq \frac{r}{R}
$$

where $0<r<R<1$. However, strict inequality in (5.21) is equivalent to the analyticity of $f(z)$ in $G_{\rho}$, for some $\rho$ with $R<\rho<1$, by virtue of Theorem 3.4. Thus, $f(z)$ has a singularity on $S_{R}$ if and only if equality holds in (5.21). 
Proof of Proposition 3.6. Suppose that $f(z)$ is analytic in the interior of $\zeta+E$ and continuous on $\{\zeta+E\}$, where $E$ is a compact set. Then, $g(t):=f(t+\zeta)$ is analytic in the interior of $E$ and continuous on $E$, which implies by hypothesis that $g(t)$ can be uniformly approximated on $E$ by $\left\{e^{-n t} P_{n}(t)\right\}_{n=0}^{\infty}$. Thus, with $z=t+\zeta, f(z)$ can be approximated on $\{\zeta+E\}$ by the weighted polynomials

$$
e^{-n(z-\zeta)} P_{n}(z-\zeta)=e^{-n z}\left(e^{n \zeta} P_{n}(z-\zeta)\right), n \geq 0 .
$$

Proof of Theorem 3.7. Because of Proposition 3.6, we may assume that the domain $H$ is such that $\bar{G} \subset H$. Further, assume to the contrary, that, for some $f(z) \neq \equiv$ which is analytic in $H$, there exists a sequence of polynomials $\left\{P_{n}(z)\right\}_{n=0}^{\infty}$, $\operatorname{deg} P_{n} \leq$ $n$, such that

$$
\lim _{n \rightarrow \infty}\left\|f(z)-e^{-n z} P_{n}(z)\right\|_{\bar{G}}=0 .
$$

It follows that

$$
\lim _{n \rightarrow \infty}\left\|e^{-n z} P_{n}(z)\right\|_{\bar{G}}=\|f\|_{\bar{G}} \neq 0 .
$$

But Corollary 4.2, for the case $r=1$, and (5.23) immediately give that

$$
\lim _{n \rightarrow \infty}\left|e^{-n z} P_{n}(z)\right|=0, \quad \text { for any } z \in \Omega_{0},
$$

where the convergence in (5.24) is locally uniform in $\Omega_{0}$. Thus, the convergence of $\left\{e^{-n z} P_{n}(z)\right\}_{n=0}^{\infty}$ to $f(z) \not \equiv 0$, locally uniformly in $H$, is impossible because $H \cap \Omega_{0} \neq$ $\emptyset$.

Proof of Theorem 3.8. Let $f(z)$ be analytic in $D_{1 / 2}:=\left\{z:|z|<\frac{1}{2}\right\}$. We shall prove that $f(z)$ can be uniformly approximated by weighted polynomials on compact subsets of $D_{1 / 2}$, using the Hermite interpolation formula. But before that, we investigate the geometric properties of the level curves of the harmonic function $u_{r}(z)$ in $\mathbb{C} \backslash \bar{D}_{r}$ (see Section 4 and Theorem 4.3), given by

$$
u_{r}(z):=U^{\mu_{\bar{D}_{r}}}(z)+Q(z), \quad 0<r<\frac{1}{2} .
$$

Note that by (4.16), we have

$$
u_{r}(z)=\operatorname{Re}\left\{\log \frac{1}{z}-\frac{r^{2}}{z}+z\right\}, \quad|z| \geq r .
$$

Thus, on defining the analytic and single-valued function

$$
g_{r}(z):=z \exp \left\{\frac{r^{2}}{z}-z\right\}, \quad|z| \geq r,
$$

we obtain that

$$
\left\{\begin{array}{l}
\left|g_{r}\left(\operatorname{Re}^{i \psi}\right)\right|=\exp \left\{\log R+\left(\frac{r^{2}}{R}-R\right) \cos \psi\right\}, \\
\arg g_{r}\left(\operatorname{Re}^{i \psi}\right)=\psi-\left(\frac{r^{2}}{R}+R\right) \sin \psi
\end{array}\right.
$$


where $R \geq r$ and where $0 \leq \psi \leq 2 \pi$. On setting $h_{r}(R, \psi):=\log R+\left(\frac{r^{2}}{R}-R\right) \cos \psi$ and recalling that $0<r<1 / 2$, it can be verified that

$$
\begin{aligned}
& \frac{\partial h_{r}(R, \psi)}{\partial R}=\frac{1}{R}-\left(\frac{r^{2}}{R^{2}}+1\right) \cos \psi>0, \\
& \frac{\partial \arg g_{r}\left(\operatorname{Re}^{i \psi}\right)}{\partial \psi}=1-\left(\frac{r^{2}}{R}+R\right) \cos \psi>0
\end{aligned}
$$

for all $\psi$ with $0 \leq \psi \leq 2 \pi$ and for all $R$ with

$$
r \leq R<\frac{1+\sqrt{1-4 r^{2}}}{2}=: \tau(r) .
$$

This implies, for each fixed $R$ with $r \leq R<\tau(r)$, that $g_{r}\left(\operatorname{Re}^{i \psi}\right)$, as a function of $\psi$, is an analytic Jordan curve in the $z$-plane, and that the Jordan curve $g_{r}\left(R^{\prime} e^{i \psi}\right)$ contains in its interior the Jordan curve $g_{r}\left(\operatorname{Re}^{i \psi}\right)$ for any $R$ and $R^{\prime}$ with $r \leq R<$ $R^{\prime}<\tau(r)$. As a consequence, $g_{r}(z)$ is than a conformal map of the annulus

$$
r \leq|z|<\tau(r) \quad(\text { for } 0<r<1 / 2) .
$$

With

$$
\hat{\rho}(r):=\tau(r) \exp \left\{\tau(r)-\frac{r^{2}}{\tau(r)}\right\},
$$

it can be verified that the level curves of $g_{r}(z)$ can be defined for

$$
\Gamma_{r, \rho}:=\left\{z \in \mathbb{C}:\left|g_{r}(\rho)\right|=\rho\right\}, \quad r \leq \rho \leq \hat{\rho}(r) .
$$

In particular, for any $r$ with $0<r<1 / 2, \Gamma_{r, r+2 \epsilon}$ is a level curve of $g_{r}(z)$, for $\epsilon>0$ sufficiently small, which contains the level curves $\Gamma_{r, r}$ and $\Gamma_{r, r+\epsilon}$. This will be used below.

Next, we interpolate $e^{n z} f(z)$ by the polynomial $P_{n}(z)$ at the $n+1$ weighted Fekete points $\left\{z_{k}^{(n+1)}\right\}_{k=1}^{n+1} \subset\{z:|z|=r\}, n=2,3, \ldots$ (cf. Section III.1 of [11]), corresponding to the weight $(4.1)$ on $\bar{D}_{r}$, where $0<r<\frac{1}{2}$. Introducing the Fekete polynomials, associated with the weight $w(z)$ [11], by

$$
\omega_{n+1}(z):=\prod_{k=1}^{n+1}\left(z-z_{k}^{(n+1)}\right),
$$

from the Hermite interpolation formula we get

$$
e^{n z} f(z)-P_{n}(z)=\frac{\omega_{n+1}(z)}{2 \pi i} \int_{\Gamma_{r, r+2 \epsilon}} \frac{f(t) e^{n t} d t}{(t-z) \omega_{n+1}(t)},
$$

where $z \in \operatorname{Int} \Gamma_{r, r+2 \epsilon}$ and where $0<r<\frac{1}{2}$. If we divide (5.34) by $e^{n z}$, then

$$
f(z)-e^{-n z} P_{n}(z)=\frac{e^{-n z} \omega_{n+1}(z)}{2 \pi i} \int_{\Gamma_{r, r+2 \epsilon}} \frac{f(t) d t}{(t-z) e^{-n t} \omega_{n+1}(t)},
$$

where $z \in \operatorname{Int} \Gamma_{r, r+2 \epsilon}$ and $0<r<\frac{1}{2}$. It is known from Theorem III.1.8 of [11] and from Theorem 4.3 that

$$
\lim _{n \rightarrow \infty}\left|\omega_{n}(z)\right|^{1 / n}=\exp \left\{-U^{\mu_{\overline{D_{r}}}}(z)\right\}
$$


locally uniformly in $|z|>r$. Thus, with $z \in \Gamma_{r, r+\epsilon}$, on estimating (5.35) by (5.36) and (5.32), we obtain

$$
\begin{aligned}
& \limsup _{n \rightarrow \infty}\left\|f(z)-e^{-n z} P_{n}(z)\right\|_{\bar{D}_{r}}^{1 / n} \leq \limsup _{n \rightarrow \infty}\left\|f(z)-e^{-n z} P_{n}(z)\right\|_{\Gamma_{r, r+\epsilon}}^{1 / n} \\
& \leq \limsup _{n \rightarrow \infty} \frac{\left\|e^{-n z} \omega_{n+1}(z)\right\|_{\Gamma_{r, r+\epsilon}}^{1 / n}}{\min _{z \in \Gamma_{r, r+2 \epsilon}}\left|e^{-n z} \omega_{n+1}(z)\right|^{1 / n}}=\frac{\left\|\exp \left\{-U^{\mu_{\overline{D_{r}}}}-Q\right\}\right\|_{\Gamma_{r, r+\epsilon}}}{\min _{z \in \Gamma_{r, r+2 \epsilon}} \exp \left\{-U^{\mu_{\bar{D}_{r}}}(z)-Q(z)\right\}} \\
& =\frac{r+\epsilon}{r+2 \epsilon}<1 .
\end{aligned}
$$

Since $r$ can be made arbitrarily close to $\frac{1}{2}$, then the approximation of $f(z)$, by the weighted polynomials $e^{-n z} P_{n}(z)$, is possible on any compact subset of $D_{1 / 2}$. The same is true for an arbitrary disk of radius $\frac{1}{2}$ by Proposition 3.6.

Suppose that $H$ contains a closed disk of radius $\frac{1}{2}$ and that there is an analytic function $f(z) \neq \equiv 0$ in $H$ which can be approximated, locally uniformly, in $H$ by weighted polynomials. By Proposition 3.6, we can shift the domain $H$, along with its enclosed disk, and we can assume that the disk of radius $\frac{1}{2}$ is now centered at the origin. Thus, we have that convergence holds for a disk of larger radius $\frac{1}{2}+\epsilon$, with some $\epsilon>0$, and that

$$
\lim _{n \rightarrow \infty}\left\|e^{-n z} P_{n}(z)\right\|_{\bar{D}_{1 / 2+\epsilon}}=\|f\|_{\bar{D}_{1 / 2+\epsilon}} \neq 0 .
$$

It follows that any sequence of weighted polynomials satisfying (5.37) must converge to 0 locally uniformly in $D_{1 / 2+\epsilon}$ by (4.28) of Corollary 4.4 , contradicting our assumption.

\section{REFERENCES}

[1] P. B. Borwein and W. Chen, Incomplete rational approximation in the complex plane, Constr. Approx. 11 (1995), 85-106. MR 95k:41024

[2] J. D. Buckholtz, A characterization of the exponential series, Amer. Math. Monthly 73, Part II (1966), 121-123. MR 34:2838

[3] R. S. Varga and A. J. Carpenter, Asymptotics for the zeros of the partial sums of $e^{z} . I I$, Computational Methods and Function Theory, Lecture Notes in Math., vol. 1435, pp. 201207, Springer-Verlag, Heidelberg, 1990. MR 92m:33004

[4] A. J. Carpenter, R. S. Varga and J. Waldvogel, Asymptotics for the zeros of the partial sums of $e^{z}$. I., Rocky Mount. J. of Math. 21 (1991), 99-119. MR 92m:33003

[5] D. Gaier, Lectures on Complex Approximation, Birkhäuser, Boston, 1987. MR 88i:30059b

[6] P. Henrici, Applied and Computational Complex Analysis, vol. 2, John Wiley and Sons, New York, 1977. MR 56:12235

[7] N. S. Landkof, Foundations of Modern Potential Theory, Springer-Verlag, Berlin, 1972. MR 50:2520

[8] G. G. Lorentz, Approximation by incomplete polynomials (problems and results), Padé and Rational Approximations: Theory and Applications (E. B. Saff and R. S. Varga, eds.), pp. 289-302, Academic Press, New York, 1977. MR 57:6956

[9] H. N. Mhaskar and E. B. Saff, The distribution of zeros of asymptotically extremal polynomials, J. Approx. Theory 65 (1991), 279-300. MR 92d:30005

[10] H. N. Mhaskar and E. B. Saff, Weighted analogues of capacity, transfinite diameter and Chebyshev constant, Constr. Approx. 8 (1992), 105-124. MR 93a:31004

[11] E. B. Saff and V. Totik, Logarithmic Potentials with External Fields, Springer-Verlag, Heidelberg, 1997.

[12] G. Szegö, Über eine Eigenshaft der Exponentialreihe, Sitzungsber. Berl. Math. Ges. 23 (1924), 50-64. 
[13] V. Totik, Weighted Approximation with Varying Weight, Lecture Notes in Math., vol. 1569, Springer-Verlag, Heidelberg, 1994. MR 96f:41002

[14] J. L. Walsh, Interpolation and Approximation by Rational Functions in the Complex Domain, Colloquium Publications, vol. 20, Amer. Math. Soc., Providence, 1969. MR 36:1572b (earlier ed.)

Institute for Computational Mathematics, Department of Mathematics and ComPuter Science, Kent State University, Kent, Ohio 44242

E-mail address: pritsker@mcs.kent.edu

Institute for Computational Mathematics, Department of Mathematics and ComPuter Science, Kent State University, Kent, Ohio 44242

E-mail address: varga@mcs.kent.edu 